\title{
Shear Wave Propagation in Multilayered Medium including an Irregular Fluid Saturated Porous Stratum with Rigid Boundary
}

\author{
Ravinder Kumar, ${ }^{1}$ Dinesh Kumar Madan, ${ }^{2}$ and Jitander Singh Sikka ${ }^{1}$ \\ ${ }^{1}$ Department of Mathematics, Maharshi Dayanand University, Rohtak, Haryana 124 001, India \\ ${ }^{2}$ Department of Mathematics, TIT\&S, Bhiwani, Haryana 127 021, India \\ Correspondence should be addressed to Dinesh Kumar Madan; dineshmadan@titsbhiwani.ac.in
}

Received 25 June 2014; Revised 5 September 2014; Accepted 8 September 2014; Published 12 November 2014

Academic Editor: Klaus Kirsten

Copyright (c) 2014 Ravinder Kumar et al. This is an open access article distributed under the Creative Commons Attribution License, which permits unrestricted use, distribution, and reproduction in any medium, provided the original work is properly cited.

\begin{abstract}
The present investigation is concerned with the study of propagation of shear waves in an anisotropic fluid saturated porous layer over a semi-infinite homogeneous elastic half-space lying under an elastic homogeneous layer with irregularity present at the interface with rigid boundary. The rectangular irregularity has been taken in the half-space. The dispersion equation for shear waves is derived by using the perturbation technique followed by Fourier transformation. Numerically, the effect of irregularity present is analysed. It is seen that the phase velocity is significantly influenced by the wave number and the depth of the irregularity. The variations of dimensionless phase velocity against dimensionless wave number are shown graphically for the different size of rectangular irregularities with the help of MATLAB.
\end{abstract}

\section{Introduction}

The earth has a layered structure, and this exerts a significant influence on the propagation of elastic waves. Inside the Earth, a very hard layer (also known as "rigid") is present. Since the composition of the Earth is heterogeneous including a very hard layer, the medium porosity and the rigid interface play significant roles in the propagation of the seismic waves. The propagation of elastic waves in homogeneous layer is of considerable importance in earthquake engineering and seismology. The study of wave propagation in elastic medium with different boundaries is of great importance to seismologists as well as to geophysicists to understand and predict the seismic behavior at different margins of earth. The propagation of shear waves has been studied by many authors assuming different forms of irregularities at the interface. Bhattacharya [1] discussed the dispersion curves for Love wave propagation in a transversely isotropic crustal layer with an irregularity in the interface. Jones [2] discussed wave propagation in a two-layered medium. Chattopadhyay [3] studied the effect of irregularities and nonhomogeneities in the crustal layer on the propagation of Love waves. Biot [4] and Deresiewcz [5] investigated the propagation of surface waves in liquid filled porous solids. Chattopadhyay et al. [6] studied the propagation of $\mathrm{SH}$ guided wave in an internal stratum with parabolic irregularity in the lower interface. Chattopadhyay and De [7] studied the dispersion equation for Love waves in a nondissipative liquid filled with porous solid underlain by an isotropic and homogeneous halfspace. They derived the dispersion equation by applying the perturbation method, and the phase velocity curve was obtained for different irregularities by using the parameters of the porous medium which were suggested by Biot [8]. Kończak [9] derived dispersion equation for shear waves in a multilayered medium including a fluid saturated porous stratum. The influence of irregularity and rigidity on the propagation of torsional waves has been discussed by Gupta et al. [10]. Love wave propagation in a porous rigid layer lying over an initially stressed half-space is discussed by Kundu et al. [11]. Madan et al. [12] also discussed the propagation of Love waves in an irregular fluid saturated porous anisotropic layer with rigid boundary. For the elastic and viscoelastic waves, a long list of references is available in the monographs of Biot [13], Lamb [14], Miklowitz [15], and Keolsky [16]. 


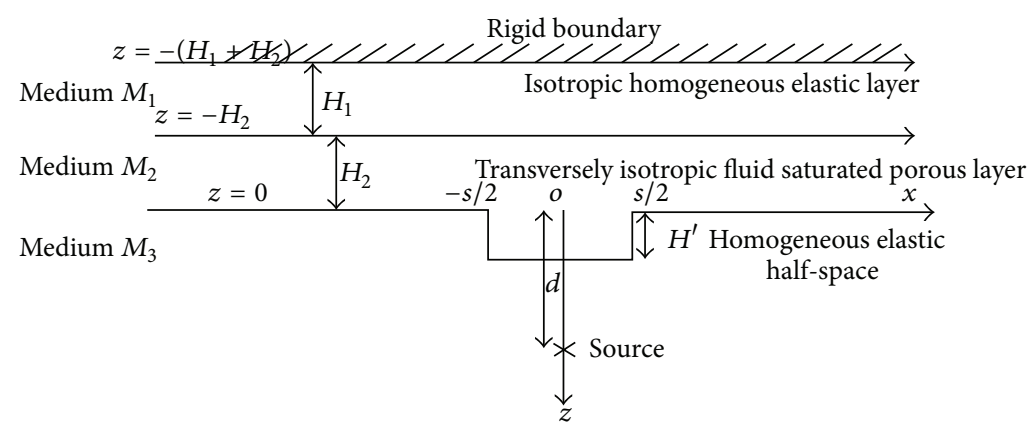

FIGURE 1: Geometry of the problem.

In this paper we have discussed the propagation of shear waves in a transversely isotropic fluid saturated porous layer resting on a homogeneous elastic half-space, lying under an elastic isotropic and homogeneous layer with rigid boundary with irregularity at the interface. The irregularity is in the form of rectangle. The dispersion curves are depicted by means of graphs for different size of irregularity and different values of common wave velocity. The influence of depth of irregularity on phase velocity and some special cases have been studied.

\section{Formulation of the Problem}

A transversely isotropic fluid saturated porous layer of thickness $\mathrm{H}_{2}$ resting on a homogeneous elastic half-space and lying under an elastic isotropic and homogeneous layer of thickness $H_{1}$ has been considered. The Cartesian coordinate system $(x, y, z)$ is chosen with $z$-axes taken vertically downward in the half-space and $x$-axes is chosen parallel to the layer in the direction of propagation of the disturbance. We assume the irregularity in the form of a rectangle with length $s$ and depth $H^{\prime}$. The origin is placed at the middle point of the interface irregularity. The source of the disturbance is placed on positive $z$-axes at a distance $d\left(d>H^{\prime}\right)$ from the origin. Therefore, the upper layer describes the medium $M_{1}$ : $-\left(H_{1}+H_{2}\right) \leq z \leq H_{2}$, the intermediate layer describes the medium $M_{2}:-H_{2} \leq z \leq 0$, and the homogeneous elastic half-space describes the medium $M_{3}: 0 \leq z<\infty$. The geometry of the problem is shown in Figure 1.

The interface between the layer and half-space is defined as

$$
z=\operatorname{ch}(x),
$$

where

$$
h(x)= \begin{cases}0 ; & x \leq-\frac{s}{2}, x \geq \frac{s}{2} \\ f(x) ; & -\frac{s}{2} \leq x \leq \frac{s}{2},\end{cases}
$$

where $\varepsilon=H^{\prime} / s$ and $\varepsilon \ll 1$.

\section{Basic Equations}

The basic equations for the medium considered are as follows.
3.1. Medium $M_{1}$. The equations of motion, without body force [17], are given by

$$
\sigma_{i j . j}^{(1)}=\rho^{(1)} \ddot{u}_{i}^{(1)},
$$

where $\sigma_{i j}^{(1)}$ are the components of stress tensor, $u_{i}^{(1)}$ are the components of displacement vector, and $\rho^{(1)}$ is the density. The comma denotes differentiation with respect to position and dot denotes differentiation with respect to time.

The constitutive relations are given by

$$
\sigma_{i j}^{(1)}=\lambda^{(1)} e_{k k}^{(1)} \delta_{i j}+2 \mu^{(1)} e_{i j}^{(1)},
$$

where $\lambda^{(1)}$ and $\mu^{(1)}$ are Lame's elastic coefficients and $\delta_{i j}$ is the Kronecker delta and

$$
2 e_{i j}^{(1)}=\left(u_{i, j}^{(1)}+u_{j, i}^{(1)}\right), \quad e_{k k}^{(1)}=u_{k, k}^{(1)}=e^{(1)} .
$$

3.2. Medium $M_{2}$. The equations of motion for the intermediate fluid saturated porous layer in the absence of body forces are [18]

$$
\begin{aligned}
& \sigma_{i j, j}^{(2)}=\rho_{11} \ddot{u}_{i}^{(2)}+\rho_{12} \ddot{U}_{i}^{(2)}-b_{i j}\left(\dot{U}_{j}^{(2)}-\dot{u}_{j}^{(2)}\right), \\
& \sigma_{, i}^{(2)}=\rho_{12} \ddot{u}_{i}^{(2)}+\rho_{22} \ddot{U}_{i}^{(2)}+b_{i j}\left(\dot{U}_{j}^{(2)}-\dot{u}_{j}^{(2)}\right),
\end{aligned}
$$

where $\sigma_{i j}^{(2)}$ are the components of stress tensor in the solid skeleton, $\sigma^{(2)}=-f p$ is the reduced pressure of the fluid ( $p$ is the pressure in the fluid, and $f$ is the porosity of the medium), and $u_{i}^{(2)}$ are the components of the displacement vector of the solid skeleton and $U_{i}^{(2)}$ are those of fluid. 
The stress-strain relations for the transverse-isotropic fluid saturated porous layer are

$$
\left[\begin{array}{c}
\sigma_{11}^{(2)} \\
\sigma_{22}^{(2)} \\
\sigma_{33}^{(2)} \\
\sigma_{23}^{(2)} \\
\sigma_{31}^{(2)} \\
\sigma_{12}^{(2)} \\
\sigma^{(2)}
\end{array}\right]
$$$$
=\left[\begin{array}{ccccccc}
2 C_{1}+C_{2} & C_{2} & C_{3} & 0 & 0 & 0 & C_{6} \\
C_{2} & 2 C_{1}+C_{2} & C_{3} & 0 & 0 & 0 & C_{6} \\
C_{3} & C_{3} & 2 C_{4} & 0 & 0 & 0 & C_{7} \\
0 & 0 & 0 & 2 C_{5} & 0 & 0 & 0 \\
0 & 0 & 0 & 0 & 2 C_{5} & 0 & 0 \\
0 & 0 & 0 & 0 & 0 & 2 C_{1} & 0 \\
C_{6} & C_{6} & C_{7} & 0 & 0 & 0 & C_{8}
\end{array}\right]\left[\begin{array}{c}
e_{11}^{(2)} \\
e_{22}^{(2)} \\
e_{33}^{(2)} \\
e_{23}^{(2)} \\
e_{31}^{(2)} \\
e_{12}^{(2)} \\
e^{(2)}
\end{array}\right],
$$

where

$$
\begin{gathered}
2 e_{i j}^{(2)}=\left(u_{i, j}^{(2)}+u_{j, i}^{(2)}\right), \\
e^{(2)}=\operatorname{div} U^{(2)} \equiv U_{j, j}^{(2)}, \\
e_{k k}=\operatorname{div} u^{(2)} \equiv u_{k, k}^{(2)},
\end{gathered}
$$

and $C_{1}, C_{2}, C_{3}, C_{4}, C_{5}, C_{6}, C_{7}$, and $C_{8}$ are the material constants.

3.3. Medium $M_{3}$. For the lower homogeneous half-space the basic equations of motion, without body force, are [17]

$$
\sigma_{i j, j}^{(3)}=\rho^{(3)} \ddot{u}_{i}^{(3)},
$$

where $\sigma_{i j, j}^{(3)}$ are the components of stress tensor, $u_{i}^{(3)}$ are the components of displacement vector, and $\rho^{(3)}$ is the density.

The constitutive relations are given by

$$
\begin{gathered}
\sigma_{i j}^{(3)}=\lambda^{(3)} e_{k k}^{(3)} \delta_{i j}+2 \mu^{(3)} e_{i j}^{(3)}, \quad 2 e_{i j}^{(3)}=\left(u_{i, j}^{(3)}+u_{j, i}^{(3)}\right), \\
e_{k k}^{(3)}=u_{k, k}^{(3)}=e^{(3)},
\end{gathered}
$$

where $\lambda^{(3)}$ and $\mu^{(3)}$ are Lame's elastic coefficients and are functions of $x, y$, and $z$.
In this paper, attention is confined to shear waves propagating in the $x y$-plane. The displacements are parallel to $y$ direction and are independent of the $y$ coordinate. Thus,

$$
\begin{aligned}
u^{(1)} \equiv w^{(1)} \equiv 0, & v^{(1)} \equiv v^{(1)}(x, z, t), \\
u^{(2)} \equiv w^{(2)} \equiv 0, & v^{(2)} \equiv v^{(2)}(x, z, t), \\
U^{(2)} \equiv W^{(2)} \equiv 0, & V^{(2)} \equiv V^{(2)}(x, z, t), \\
u^{(3)} \equiv w^{(3)} \equiv 0, & v^{(3)} \equiv v^{(3)}(x, z, t),
\end{aligned}
$$

and the equations of motion (3), (6), and (9) with the help of (4), (5), (7), (8), and (10), respectively, reduce to the form

$$
\begin{gathered}
\left\{\frac{\partial^{2}}{\partial x^{2}}+\frac{\partial^{2}}{\partial z^{2}}\right\} v^{(1)}=\frac{1}{\beta_{1}^{2}} \frac{\partial^{2} v^{(1)}}{\partial t^{2}}, \\
\left\{C_{1} \frac{\partial^{2}}{\partial x^{2}}+C_{5} \frac{\partial^{2}}{\partial z^{2}}\right. \\
\left.-\left[\rho_{11} \partial_{t}^{2}+b_{11} \partial_{t}-\frac{\left(\rho_{12} \partial_{t}^{2}-b_{11} \partial_{t}\right)^{2}}{\rho_{22} \partial_{t}^{2}+b_{11} \partial_{t}}\right]\right\} \\
\times\left(v^{(2)}, V^{(2)}\right)=0, \\
\left\{\frac{\partial^{2}}{\partial x^{2}}+\frac{\partial^{2}}{\partial z^{2}}\right\} v^{(3)}=\frac{1}{\beta_{3}^{2}} \frac{\partial^{2} v^{(3)}}{\partial t^{2}} .
\end{gathered}
$$

The appropriate boundary conditions for the considered problem are as follows.

(i) At the rigid surface $z=-\left(H_{1}+H_{2}\right)$, the displacement component vanishes; that is,

$$
v^{(1)}\left(x, z=-\left(H_{1}+H_{2}\right), t\right)=0 .
$$

(ii) At the interface $z=-H_{2}$, the displacements are continuous; that is,

$$
v^{(1)}\left(x, z=-H_{2}, t\right)=v^{(2)}\left(x, z=-H_{2}, t\right) .
$$

(iii) At the interface $z=-H_{2}$, the shear stress components are continuous; that is,

$$
\sigma_{32}^{(1)}\left(x, z=-H_{2}, t\right)=\sigma_{32}^{(2)}\left(x, z=-H_{2}, t\right) .
$$

(iv) At the interface $z=\varepsilon h(x)$, the displacements are continuous; that is,

$$
v^{(2)}(x, z=\operatorname{ch}(x), t)=v^{(3)}(x, z=\operatorname{ch}(x), t) .
$$

(v) The stresses are continuous at the interface $z=\operatorname{ch}(x)$; that is,

$$
\begin{aligned}
C_{5} \frac{\partial v^{(2)}}{\partial z}-C_{1} \varepsilon h^{\prime}(x) \frac{\partial v^{(2)}}{\partial x} & =\mu\left(\frac{\partial v^{(3)}}{\partial z}-\varepsilon h^{\prime}(x) \frac{\partial v^{(3)}}{\partial x}\right), \\
h^{\prime}(x) & =\frac{d h(x)}{d x} .
\end{aligned}
$$

Thus (12)-(14) with the previous boundary conditions are the governing equations of the problem considered. 


\section{Solution of the Problem}

For waves changing harmonically with time $t$ and propagating in $x$-direction, we obtain

$$
\begin{aligned}
v^{(1)}(z, x, t) & =v_{0}^{(1)}(z, x) \exp (i \omega t), \\
v^{(2)}(z, x, t) & =v_{0}^{(2)}(z, x) \exp (i \omega t), \\
V^{(2)}(z, x, t) & =V_{0}^{(2)}(z, x) \exp (i \omega t), \\
v^{(3)}(z, x, t) & =v_{0}^{(3)}(z, x) \exp (i \omega t),
\end{aligned}
$$

where $\omega$ is the angular frequency.

Thus equations of motion (12)-(14) take the form of

$$
\begin{gathered}
\frac{\partial^{2} v_{0}^{(1)}}{\partial x^{2}}+\frac{\partial^{2} v_{0}^{(1)}}{\partial z^{2}}+\frac{\omega^{2}}{\beta_{1}^{2}} v_{0}^{(1)}=0, \\
\left(C_{1} \frac{\partial^{2}}{\partial x^{2}}+C_{5} \frac{\partial^{2}}{\partial z^{2}}+\xi_{1}^{2}\right)\left(v_{0}^{(2)}, V_{0}^{(2)}\right)=0, \\
\frac{\partial^{2} v_{0}^{(3)}}{\partial x^{2}}+\frac{\partial^{2} v_{0}^{(3)}}{\partial z^{2}}+\frac{\omega^{2}}{\beta_{3}^{2}} v_{0}^{(3)}=0,
\end{gathered}
$$

where $\xi_{1}^{2}=\alpha_{1}+i \alpha_{2}, \alpha_{1}$ and $\alpha_{2}$ are given in Appendix section.

Define Fourier transformation $\bar{v}_{0}^{(1)}(z, \eta)$ of $v_{0}^{(1)}(z, \eta)$ as

$$
\bar{v}_{0}^{(1)}(z, \eta)=\int_{-\infty}^{\infty} v_{0}^{(1)}(z, x) e^{i \eta x} d x .
$$

And inverse Fourier transformation is given by

$$
v_{0}^{(1)}(z, x)=\frac{1}{2 \pi} \int_{-\infty}^{\infty} \bar{v}_{0}^{(1)}(z, \eta) e^{-i \eta x} d \eta
$$

and so forth.

The Fourier transformation of (21) then is

$$
\begin{aligned}
& \frac{\partial^{2} \bar{v}_{0}^{(1)}}{\partial z^{2}}+\chi_{1}^{2} \bar{v}_{0}^{(1)}=0, \\
& \frac{\partial^{2} \bar{v}_{0}^{(2)}}{\partial z^{2}}+\chi_{2}^{2} \bar{v}_{0}^{(2)}=0, \\
& \frac{\partial^{2} \bar{V}_{0}^{(2)}}{\partial z^{2}}+\chi_{2}^{2} \bar{V}_{0}^{(2)}=0, \\
& \frac{\partial^{2} \bar{v}_{0}^{(3)}}{\partial z^{2}}-\chi_{3}^{2} \bar{v}_{0}^{(3)}=0,
\end{aligned}
$$

where

$$
\begin{gathered}
\chi_{1}^{2}=\left(\frac{\omega^{2}}{\beta_{1}^{2}}-\eta^{2}\right), \\
\chi_{2}^{2}=\frac{C_{1}}{C_{5}}\left(\frac{\xi_{1}^{2}}{C_{1}}-\eta^{2}\right), \\
\chi_{3}^{2}=\left(\eta^{2}-\frac{\omega^{2}}{\beta_{3}^{2}}\right) .
\end{gathered}
$$

The solutions of (24) are

$$
\begin{gathered}
\bar{v}_{0}^{(1)}=A \cos \chi_{1} z+B \sin \chi_{1} z, \\
\bar{v}_{0}^{(2)}=C \cos \chi_{2} z+D \sin \chi_{2} z, \\
\bar{V}_{0}^{(2)}=\bar{C} \cos \chi_{2} z+\bar{D} \sin \chi_{2} z, \\
\bar{v}_{0}^{(3)}=E \exp \left(-\chi_{3} z\right),
\end{gathered}
$$

where $A, B, \bar{A}, \bar{B}$, and $D$ are functions of $\eta$.

Thus, by inverse Fourier transformation, we obtain

$$
\begin{aligned}
& v_{0}^{(1)}(z, x)=\frac{1}{2 \pi} \int_{-\infty}^{\infty}\left(A \cos \chi_{1} z+B \sin \chi_{1} z\right) e^{-i \eta x} d \eta, \\
& v_{0}^{(2)}(z, x)=\frac{1}{2 \pi} \int_{-\infty}^{\infty}\left(C \cos \chi_{2} z+D \sin \chi_{2} z\right) e^{-i \eta x} d \eta, \\
& V_{0}^{(2)}(z, x)=\frac{1}{2 \pi} \int_{-\infty}^{\infty}\left(\bar{C} \cos \chi_{2} z+\bar{D} \sin \chi_{2} z\right) e^{-i \eta x} d \eta, \\
& v_{0}^{(3)}(z, x)=\frac{1}{2 \pi} \int_{-\infty}^{\infty}\left(E e^{-\chi_{3} z}+\frac{2}{\chi_{3}} e^{\chi_{3} z} e^{-\chi_{3} d}\right) e^{-i \eta x} d \eta,
\end{aligned}
$$

where the second term in the integrand of $v_{0}^{(3)}(z, x)$ is introduced due to the source in the lower half-space [19].

The relations between the constants $\bar{C}, \bar{D}$ and $C, D$ are provided by (13).

We set the following approximations due to small value of $\varepsilon$ :

$$
\begin{gathered}
A \cong A_{0}+A_{1} \varepsilon, \quad B \cong B_{0}+B_{1} \varepsilon, \\
C \cong C_{0}+C_{1} \varepsilon, \quad D \cong D_{0}+D_{1} \varepsilon, \quad E \cong E_{0}+E_{1} \varepsilon .
\end{gathered}
$$

Since the boundary is not uniform, the terms $A, B, C, D$, and $E$ in (30) are also functions of $\varepsilon$. Expanding these terms in ascending powers of $\varepsilon$ and keeping in view that $\varepsilon$ is small and so retaining the terms up to the first order of $\varepsilon, A, B$, $C, D$, and $E$ can be approximated as in (30). In physical situations, when the depth $H^{\prime}$ of the irregular boundary is too small with respect to the length of the boundary $s$, the above assumptions are justified. Further, for small $\varepsilon$,

$$
e^{ \pm \alpha \varepsilon h} \cong 1 \pm \alpha \varepsilon h, \quad \cos \chi_{1} \varepsilon h \cong 1, \quad \sin \chi_{1} \varepsilon h \cong \chi_{1} \varepsilon h,
$$

where $\alpha$ is any quantity.

Define Fourier transformation of $h(x)$ as

$$
\bar{h}(\lambda)=\int_{-\infty}^{\infty} h(x) e^{i \lambda x} d x
$$

and the inverse Fourier transformation is

$$
h(x)=\frac{1}{2 \pi} \int_{-\infty}^{\infty} \bar{h}(\lambda) e^{-i \lambda x} d \lambda .
$$

Therefore,

$$
h^{\prime}(x)=\frac{-i}{2 \pi} \int_{-\infty}^{\infty} \lambda \bar{h}(\lambda) e^{-i \lambda x} d \lambda
$$


Now, by using boundary conditions (15)-(19) along with (27) and (29)-(30) we obtain a system of ten equations (after equating the absolute term (terms not containing $\varepsilon$ ) and the coefficients of $\varepsilon$ ):

$$
\begin{gathered}
A_{0} \cos \left(H_{1}+H_{2}\right) \chi_{1}-B_{0} \sin \left(H_{1}+H_{2}\right) \chi_{1}=0 \\
A_{0} \cos H_{2} \chi_{1}-B_{0} \sin H_{2} \chi_{1}-C_{0} \cos H_{2} \chi_{2}+D_{0} \sin H_{2} \chi_{2} \\
=0, \\
\mu \chi_{1}\left[A_{0} \sin H_{2} \chi_{1}+B_{0} \cos H_{2} \chi_{1}\right] \\
-C_{5} \chi_{2}\left[C_{0} \sin H_{2} \chi_{2}+D_{0} \cos H_{2} \chi_{2}\right]=0 \\
\chi_{3}\left(C_{0}-E_{0}\right)=2 e^{-\chi_{3} d}, \\
\mu \chi_{3} E_{0}+C_{5} \chi_{2} D_{0}=2 \mu e^{-\chi_{3} d}, \\
A_{1} \cos \left(H_{1}+H_{2}\right) \chi_{1}-B_{1} \sin \left(H_{1}+H_{2}\right) \chi_{1}=0 \\
A_{1} \cos H_{2} \chi_{1}-B_{1} \sin H_{2} \chi_{1} \\
-C_{1} \cos H_{2} \chi_{2}+D_{1} \sin H_{2} \chi_{2}=0 \\
\left.\sin H_{2} \chi_{1}+B_{1} \cos H_{2} \chi_{1}\right] \\
-C_{5} \chi_{2}\left[C_{1} \sin H_{2} \chi_{2}+D_{1} \cos H_{2} \chi_{2}\right]=0 \\
E_{1}-C_{1}=R_{1}(k) \\
\mu \chi_{3} E_{1}+C_{5} \chi_{2} D_{1}=R_{2}(k)
\end{gathered}
$$

where $R_{1}(k)$ and $R_{2}(k)$ are given in Appendix section.

Solve the system of equations for $A_{0}, B_{0}, C_{0}, D_{0}, E_{0}, A_{1}$, $B_{1}, C_{1}, D_{1}$, and $E_{1}$ and the corresponding values are given in Appendix section.

The displacement in the anisotropic layer is

$$
\begin{aligned}
v_{0}^{(2)}=\frac{1}{2 \pi} \int_{-\infty}^{\infty} \frac{4 \mu e^{-\chi_{3} d}}{E(k)}\left[1+\frac{\varepsilon\left(R_{2}-\mu \chi_{3} R_{1}\right) e^{\chi_{3} d}}{4 \mu}\right] \\
\times\left[B_{2}+B_{3}\right] e^{-i k x} d k,
\end{aligned}
$$

where $B_{2}$ and $B_{3}$ are given in Appendix section.

Now from (1), (2), and (32), we have

$$
\bar{h}(\lambda)=\frac{2 s}{\lambda} \sin \frac{\lambda s}{2}
$$

Using values of $R_{1}(k)$ and $R_{2}(k)$ as given in Appendix section, we obtain

$$
\left(R_{2}-\mu \chi_{3} R_{1}\right)=\frac{s}{\pi} \int_{-\infty}^{\infty}[\phi(k-\lambda)+\phi(k+\lambda)] \frac{1}{\lambda} \sin \frac{\lambda s}{2} d \lambda,
$$

where $\phi(k-\lambda)$ is given in Appendix section.
Using asymptotic formula of Willis [19] and Tranter [20] and neglecting the terms containing $2 / s$ and highest powers of $2 / s$ for large $s$, we obtain

$$
\begin{gathered}
\int_{-\infty}^{\infty}[\phi(k-\lambda)+\phi(k+\lambda)] \frac{1}{\lambda} \sin \frac{\lambda s}{2} d \lambda \\
\cong \frac{\pi}{2} \cdot 2 \phi(k)=\pi \phi(k) .
\end{gathered}
$$

Now using (38) and (39), we obtain

$$
R_{2}-\mu \chi_{3} R_{1}=s \phi(k)=\frac{H^{\prime}}{\varepsilon} \phi(k) .
$$

Therefore the displacement in the anisotropic layer is

$$
v_{0}^{(2)}=\frac{1}{2 \pi} \int_{-\infty}^{\infty} \frac{4 \mu e^{-\chi_{3} d}}{E(k)\left[1-H^{\prime} \psi(k) e^{\chi_{3} d}\right]}\left[B_{2}+B_{3}\right] e^{-i k x} d k
$$

where $\psi(k)=\phi(k) / 4 \mu$.

The value of this integral depends entirely on the contribution of the poles of the integrand. The poles are located at the roots of the equation:

$$
E(k)\left\{1-H^{\prime} \psi(k) e^{\chi_{3} d}\right\}=0 .
$$

This equation is the dispersion equation for $\mathrm{SH}$ waves.

If $c$ is the common wave velocity of wave propagating along the surface, then we can set in $(42 a) \omega=c k(\omega$ is the circular frequency and $k$ is the wave number), $\chi_{1}=P_{1} k$, $\chi_{2}=P_{2} k$, and $\chi_{3}=P_{3} k$ where

$$
\begin{gathered}
P_{1}=\sqrt{\frac{c^{2}}{\beta_{1}^{2}}-1,} \\
P_{2}=\sqrt{\left(\frac{1}{C_{5}}\left(\frac{c^{2}}{c_{G}^{2}} \cdot F(\omega)-C_{1}\right)+i \frac{1}{C_{5}} \cdot \frac{c^{2}}{c_{G}^{2}} \cdot R(\omega)\right)}, \\
P_{3}=\sqrt{1-\frac{c^{2}}{\beta_{3}^{2}}} .
\end{gathered}
$$

Solving (42a), we obtain

$$
\begin{aligned}
\frac{\mu P_{1}}{C_{5} P_{2}}\left\{\left(1+\tan P_{1} k H_{2} \tan P_{1} k\left(H_{1}+H_{2}\right)\right)\right. \\
\times\left[\left(1-P_{3} k H^{\prime}\right)\left(C_{5} P_{2}+\mu P_{3} \tan P_{2} k H_{2}\right)\right. \\
\left.\left.\quad-H^{\prime} P_{2} k\left(C_{5} P_{2} \tan P_{2} k H_{2}-\mu P_{3}\right)\right]\right\} \\
=\left\{\left(\tan P_{1} k\left(H_{1}+H_{2}\right)-\tan P_{1} k H_{2}\right)\right. \\
\quad \times\left[\left(1-P_{3} k H^{\prime}\right)\left(C_{5} P_{2} \tan P_{2} k H_{2}-\mu P_{3}\right)\right. \\
\left.\left.\quad+H^{\prime} P_{2} k\left(C_{5} P_{2}-\mu P_{3} \tan P_{2} k H_{2}\right)\right]\right\} .
\end{aligned}
$$

Since the quantity $P_{2}^{2}$ is complex, so we have

$$
P_{2}=k_{1}+i k_{2}
$$




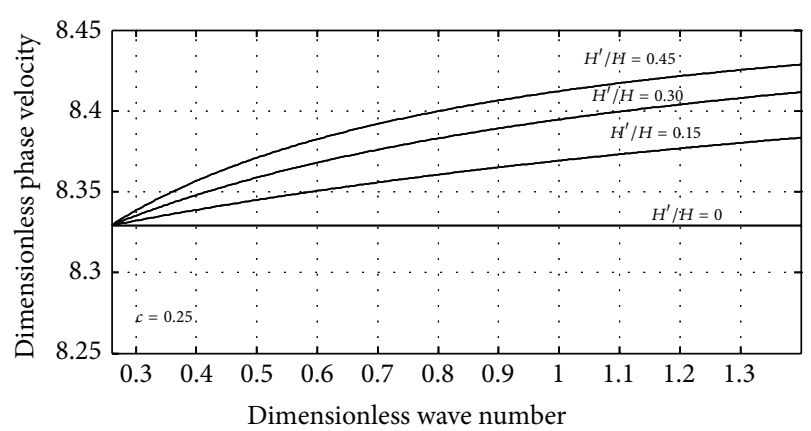

Figure 2

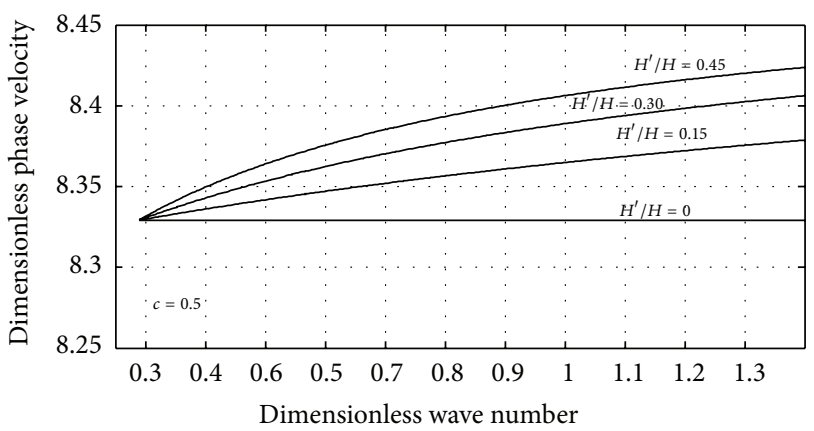

FIGURE 3

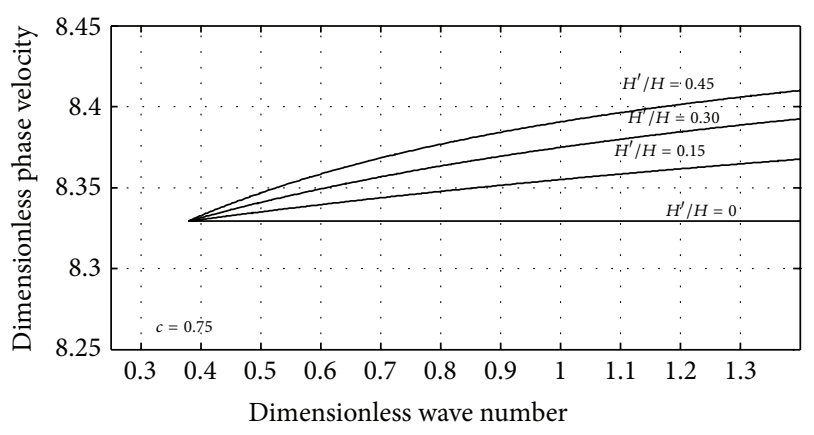

FIgURe 4

where $k_{1}$ and $k_{2}$ are the real and imaginary parts of $P_{2}$ and are given in Appendix section.

Equation (43) is complex and its real part gives the dispersion equation for shear waves.

\section{Numerical Results and Discussions}

In order to investigate the effect of irregularity present in the transversely isotropic fluid saturated porous layer and to compare the results numerically between the phase velocity and the wave number, we will use the values of elastic constants given by Ding et al. [21] for medium $M_{2}$ and by Kończak [9] for media $M_{1}$ and $M_{3}$. And by using MATLAB, we obtain the following graph for different values of common wave velocity $c$ for two special cases.

Case I. When $\mathrm{H}_{2}=0$ which is the wave propagation in elastic homogeneous layer lying over a homogeneous half-space, the

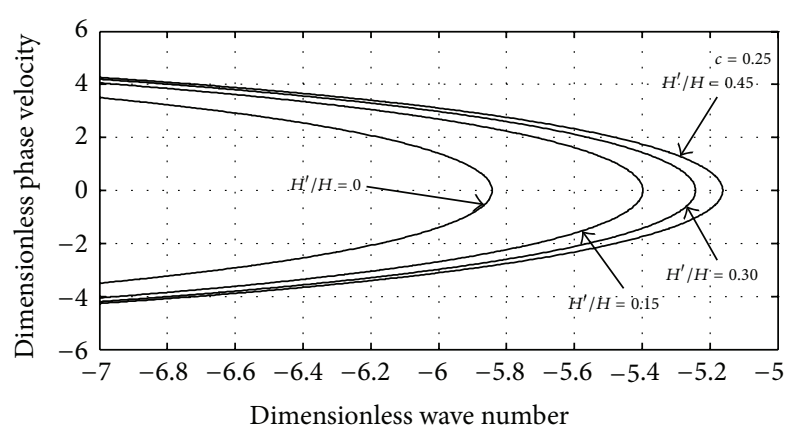

Figure 5

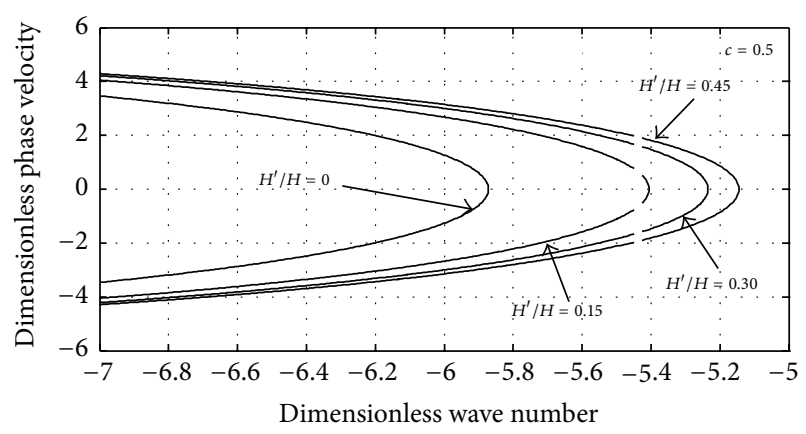

Figure 6

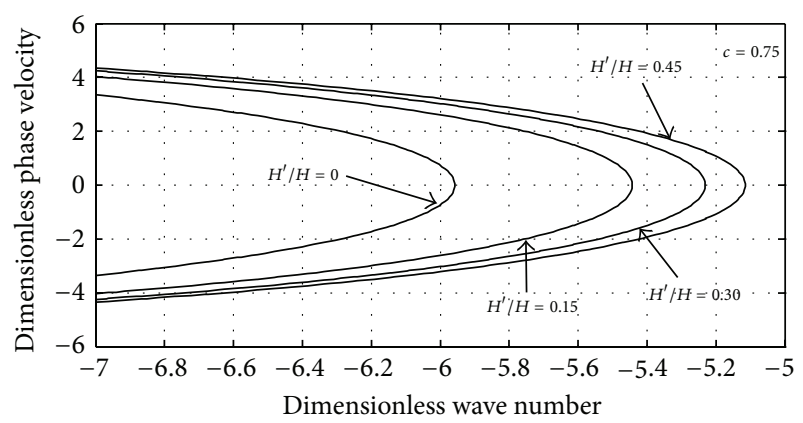

FIGURE 7

variations of the dimensionless phase velocity $\left(c / c_{G}\right)$ against the dimensionless wave number $(\mathrm{kH})$ in an elastic isotropic homogeneous layer over a homogeneous elastic half-space for different values of $H^{\prime} / H(0,0.15,0.30,0.45)$ and $c=0.25, c=$ 0.5 , and $c=0.75$ are shown in Figures 2, 3, and 4 .

Case II. When $H_{1}=0$ which is the wave propagation in a transversely isotropic fluid saturated porous layer lying over a homogeneous half-space, the variations of the dimensionless phase velocity $\left(c / c_{G}\right)$ against the dimensionless wave number $(\mathrm{kH})$ in a transversely isotropic fluid saturated porous layer over a homogeneous elastic half-space for different values of $H^{\prime} / H(0,0.15,0.30,0.45)$ and $c=0.25, c=0.5$, and $c=0.75$ are shown in Figures 5, 6, and 7.

The dimensionless phase velocity $\left(c / c_{G}\right)$ is plotted against the dimensionless wave number $(\mathrm{kH})$ in Figures 2, 3, 4, 5, 
6 , and 7 . We conclude that the multilayered medium with irregularity and rigid boundary have significant effect on the propagation of shear waves, and the phase velocity in a layer with irregularity is affected by not only the shape of irregularity but also the wave number, the ratio of the depth of the irregularity to layer width and layer structure.

\section{Appendix}

Consider the following:

$$
\begin{gathered}
\alpha_{1}=\frac{F \omega^{2}}{c_{G}^{2}}, \quad \alpha_{2}=\frac{R \omega^{2}}{c_{G}^{2}}, \\
F=F(\omega)=\frac{1+\Omega^{2} \gamma_{22} C^{\prime}}{1+\left(\Omega \gamma_{22}\right)^{2}} \cdot \frac{\gamma_{22}}{C^{\prime}}, \\
R=R(\omega)=\frac{\left(C^{\prime}-\gamma_{22}\right) \Omega}{1+\left(\Omega \gamma_{22}\right)^{2}} \cdot \frac{\gamma_{22}}{C^{\prime}}, \\
C^{\prime}=\gamma_{11} \gamma_{22}-\gamma_{12}^{2}, \quad \gamma_{k l}=\frac{\rho_{k l}}{\rho} \quad(k, l=1,2), \\
c_{G}^{2}=\left(\rho_{11}-\frac{\rho_{12}^{2}}{\rho_{22}}\right)^{-1}, \quad \Omega=\frac{\rho \omega}{b_{11}},
\end{gathered}
$$

where $\Omega$ is the dimensionless frequency and $c_{G}$ is the velocity of shear wave in the porous layer.

Consider the following:

$$
\begin{aligned}
R_{1}(k)=\frac{1}{2 \pi} \int_{-\infty}^{\infty} & {\left[\left(D_{0} \chi_{2}+E_{0} \chi_{3}-2 e^{-\chi_{3} d}\right)\right]^{\eta=k-\lambda} \bar{h}(\lambda) d \lambda, } \\
R_{2}(k)=\frac{1}{2 \pi} \int_{-\infty}^{\infty}[ & \left\{C_{5} \chi_{2}^{2} C_{0}-\mu\left(\chi_{3}^{2} E_{0}+2 \chi_{3} e^{-\chi_{3} d}\right)\right\} \\
& \left.-\lambda k\left\{C_{0} C_{1}+\mu\left(E_{0}+\frac{2}{\chi_{3}} e^{-\chi_{3} d}\right)\right\}\right]^{\eta=k-\lambda}
\end{aligned}
$$$$
\times \bar{h}(\lambda) d \lambda
$$$$
A_{0}=\frac{4 \mu e^{-\chi_{3} d} C_{5} \chi_{2}\left(1+\tan ^{2} \chi_{2} H_{2}\right) \tan \chi_{1}\left(H_{1}+H_{2}\right)}{E(k)},
$$$$
B_{0}=\frac{4 \mu e^{-\chi_{3} d} C_{5} \chi_{2}\left(1+\tan ^{2} \chi_{2} H_{2}\right)}{E(k)},
$$

$$
\begin{aligned}
C_{0}= & 4 \mu e^{-\chi_{3} d} \\
& \times\left[\mu \chi_{1} \tan \chi_{2} H_{2}\left(1+\tan \chi_{1} H_{2} \tan \chi_{1}\left(H_{1}+H_{2}\right)\right)\right. \\
& \left.\quad+C_{5} \chi_{2}\left(\tan \chi_{1} H_{2}-\tan \chi_{1}\left(H_{1}+H_{2}\right)\right)\right] \\
& \times(E(k))^{-1},
\end{aligned}
$$$$
D_{0}=4 \mu e^{-\chi_{3} d}
$$$$
\times\left[\mu \chi_{1}\left(1+\tan \chi_{1} H_{2} \tan \chi_{1}\left(H_{1}+H_{2}\right)\right)\right.
$$$$
\left.-C_{5} \chi_{2} \tan \chi_{2} H_{2}\left(\tan \chi_{1}\left(H_{1}+H_{2}\right)-\tan \chi_{1} H_{2}\right)\right]
$$$$
\times(E(k))^{-1} \text {, }
$$$$
E_{0}=\frac{2 e^{-\chi_{3} d}}{\chi_{3} E(k)}
$$$$
\times\left[\left\{\mu \chi_{1}\left(1+\tan \chi_{1} H_{2} \tan \chi_{1}\left(H_{1}+H_{2}\right)\right)\right.\right.
$$$$
\left.\times\left(\mu \chi_{3} \tan \chi_{2} H_{2}-C_{5} \chi_{2}\right)\right\}
$$$$
+\left\{C_{5} \chi_{2}\left(\tan \chi_{1}\left(H_{1}+H_{2}\right)-\tan \chi_{1} H_{2}\right)\right.
$$$$
\left.\left.\times\left(C_{5} \chi_{2} \tan \chi_{2} H_{2}+\mu \chi_{3}\right)\right\}\right],
$$$$
A_{1}=\left(\left(R_{2}-\mu \chi_{3} R_{1}\right) C_{5} \chi_{2}\right.
$$$$
\left.\times\left(1+\tan ^{2} \chi_{2} H_{2}\right) \tan \chi_{1}\left(H_{1}+H_{2}\right)\right)
$$$$
\times(E(k))^{-1},
$$$$
B_{1}=\frac{\left(R_{2}-\mu \chi_{3} R_{1}\right) C_{5} \chi_{2}\left(1+\tan ^{2} \chi_{2} H_{2}\right)}{E(k)},
$$

$$
C_{1}=\left(R_{2}-\mu \chi_{3} R_{1}\right)
$$

$$
\begin{aligned}
\times\left[\mu \chi_{1} \tan \chi_{2} H_{2}\left(1+\tan \chi_{1} H_{2} \tan \chi_{1}\left(H_{1}+H_{2}\right)\right)\right. \\
\left.+C_{5} \chi_{2}\left(\tan \chi_{1} H_{2}-\tan \chi_{1}\left(H_{1}+H_{2}\right)\right)\right] \\
\times(E(k))^{-1} \\
D_{1}=\left(R_{2}-\mu \chi_{3} R_{1}\right) \\
\times\left[\mu \chi_{1}\left(1+\tan \chi_{1} H_{2} \tan \chi_{1}\left(H_{1}+H_{2}\right)\right)\right. \\
\quad-C_{5} \chi_{2} \tan \chi_{2} H_{2} \\
\left.\quad \times\left(\tan \chi_{1}\left(H_{1}+H_{2}\right)-\tan \chi_{1} H_{2}\right)\right] \\
\quad \times(E(k))^{-1},
\end{aligned}
$$$$
E_{1}=\frac{1}{E(k)}
$$

$$
\begin{aligned}
\times & {\left[\left\{\mu \chi_{1}\left(R_{2} \tan \chi_{2} H_{2}+C_{5} \chi_{2} R_{1}\right)\right.\right.} \\
& \left.\times\left(1+\tan \chi_{1} H_{2} \tan \chi_{1}\left(H_{1}+H_{2}\right)\right)\right\} \\
& +\left\{C_{5} \chi_{2}\left(R_{2}-C_{5} \chi_{2} R_{1} \tan \chi_{2} H_{2}\right)\right. \\
& \left.\left.\times\left(\tan \chi_{1}\left(H_{1}+H_{2}\right)-\tan \chi_{1} H_{2}\right)\right\}\right],
\end{aligned}
$$


where

$$
\begin{gathered}
E(k)=\left[\left\{\mu \chi_{1}\left(1+\tan \chi_{1} H_{2} \tan \chi_{1}\left(H_{1}+H_{2}\right)\right)\right.\right. \\
\left.\times\left(\mu \chi_{3} \tan \chi_{2} H_{2}+C_{5} \chi_{2}\right)\right\} \\
-\left\{C_{5} \chi_{2}\left(\tan \chi_{1}\left(H_{1}+H_{2}\right)-\tan \chi_{1} H_{2}\right)\right. \\
\left.\left.\times\left(C_{5} \chi_{2} \tan \chi_{2} H_{2}-\mu \chi_{3}\right)\right\}\right], \\
B_{2}=\mu \chi_{1}\left\{\left(1+\tan \chi_{1} H_{2} \tan \chi_{1}\left(H_{1}+H_{2}\right)\right)\right. \\
\left.\times\left(\tan \chi_{2} H_{2} \cos \chi_{2} z+\sin \chi_{2} z\right)\right\}, \\
B_{3}=C_{5} \chi_{2}\left\{\left(\tan \chi_{1}\left(H_{1}+H_{2}\right)-\tan \chi_{1} H_{2}\right)\right. \\
\left.\times\left(\cos \chi_{2} z-\tan \chi_{2} H_{2} \sin \chi_{2} z\right)\right\}, \\
\phi(k-\lambda)=A_{2}+A_{3}, \\
A_{2}=C_{5} \chi_{2}^{2} C_{0}-\mu \chi_{3}\left(\chi_{2} D_{0}-4 e^{-\chi_{3} d}\right), \\
A_{3}=-\lambda k\left[C_{1} C_{0}+\mu\left(E_{0}+\frac{2}{\chi_{3}} e^{-\chi_{3} d}\right)\right] .
\end{gathered}
$$

When $z^{2}=x+i y$ then

$$
z=\left\{\frac{1}{2}\left(\sqrt{x^{2}+y^{2}}+y\right)\right\}^{1 / 2}+i\left\{\frac{1}{2}\left(\sqrt{x^{2}+y^{2}}-y\right)\right\}^{1 / 2}
$$

therefore, from (42b) and (44),

$$
\begin{aligned}
k_{1}=\{ & \frac{1}{2} \\
& \times\left(\left(\left\{\frac{1}{C_{5}}\left(\frac{c^{2}}{c_{G}^{2}} \cdot F(\omega)-C_{1}\right)\right\}^{2}\right.\right. \\
& \left.+\left(\frac{1}{C_{5}} \cdot \frac{c^{2}}{c_{G}^{2}} \cdot R(\omega)\right)^{2}\right)^{1 / 2} \\
& \left.\left.+\left\{\frac{1}{C_{5}}\left(\frac{c^{2}}{c_{G}^{2}} \cdot F(\omega)-C_{1}\right)\right\}\right)\right\}^{1 / 2} \\
k_{2}=\left\{\frac{1}{2}\right. & \times\left(\left(\left\{\frac{1}{C_{5}}\left(\frac{c^{2}}{c_{G}^{2}} \cdot F(\omega)-C_{1}\right)\right\}^{2}\right.\right. \\
& \left.\left.+\left(\frac{1}{C_{5}} \cdot \frac{c^{2}}{c_{G}^{2}} \cdot R(\omega)\right)^{2}\right)\right\}^{1 / 2} \\
& \left.\left.-\left\{\frac{1}{C_{5}}\left(\frac{c^{2}}{c_{G}^{2}} \cdot F(\omega)-C_{1}\right)\right\}\right)\right\}^{1 / 2} .
\end{aligned}
$$

\section{Conflict of Interests}

The authors declare that there is no conflict of interests regarding the publication of this paper.

\section{Acknowledgments}

The authors are grateful to the reviewers and Editor Klaus Kirsten for constructive suggestions for the improvement of this paper.

\section{References}

[1] J. Bhattacharya, "On the dispersion curve for Love wave due to irregularity in the thickness of the transversely isotropic crustal layer," Gerlands Beiträge zur Geophysik, vol. 6, pp. 324-334, 1962.

[2] J. P. Jones, "Wave propagation in a two-layered medium," Journal of Applied Mechanics, vol. 31, pp. 213-222, 1964.

[3] A. Chattopadhyay, "On the dispersion equation for Love wave due to irregularity in the thickness of the non-homogeneous crustal layer," Acta Geophysica Polonica, vol. 23, pp. 307-317, 1975.

[4] M. A. Biot, "Propagation of elastic waves in liquid filled porous solid," Journal of Applied Physics, vol. 27, pp. 459-467, 1956.

[5] H. Deresiewicz, "The effect of boundaries on wave propagation in a liquid-filled porous solid, I: reflection of plane waves at a free plane boundary (non-dissipative case)," Bulletin of the Seismological Society of America, vol. 50, pp. 599-607, 1960.

[6] A. Chattopadhyay, M. Chakraborty, and A. K. Pal, "Effects of irregularity on the propagation of guided SHwaves," Journal de Mecanique Theorique et Appliquee, vol. 2, no. 2, pp. 215-225, 1983.

[7] A. Chattopadhyay and R. K. De, "Love type waves in a porous layer with irregular interface," International Journal of Engineering Science, vol. 21, no. 11, pp. 1295-1303, 1983.

[8] M. A. Biot, Mechanics of Incremental Deformation, John Wiley \& Sons, New York, NY, USA, 1961.

[9] Z. Kończak, "On propagation of shear waves in a multilayer medium including a fluid-saturated porous stratum," Acta Mechanica, vol. 79, no. 3-4, pp. 169-181, 1989.

[10] S. Gupta, A. Chattopadhyay, and S. Kundu, "Influence of irregularity and rigidity on the propagation of torsional wave," Applied Mathematical Sciences, vol. 4, no. 17-20, pp. 805-816, 2010.

[11] S. Kundu, S. Gupta, and D. K. Majhi, "Love wave propagation in porous rigid layer lying over an initially stressed half space," Applied Physics \& Mathematics, vol. 3, no. 2, pp. 140-142, 2013.

[12] D. K. Madan, R. Kumar, and J. S. Sikka, "Love wave propagation in an irregular fluid saturated porous anisotropic layer with rigid boundary," Journal of Applied Sciences Research, vol. 10, no. 4, pp. 281-287, 2014.

[13] M. A. Biot, "Generalized theory of acoustic propagation in porous dissipative media," Acoustical Society of America, vol. 34, pp. 1254-1264, 1962.

[14] H. Lamb, "On waves in elastic plate," Proceedings of the Royal Society of London A, vol. 93, pp. 114-128, 1926.

[15] J. Miklowitz, "Elastic wave propagation," in Applied Mechanics Surveys, pp. 830-836, Spartan Books, Washington, DC, USA, 1966.

[16] H. Keolsky, "The propagation of stress waves in viscoelastic solids," in Applied Mechanics Surveys, Spartan Books, 1966. 
[17] W. M. Ewing, W. S. Jardetzky, and F. Press, Elastic Waves in Layered Media, McGraw-Hill, London, UK, 1957.

[18] M. A. Biot, "Theory of elasticity and consolidation for a porous anisotropic solid," Journal of Applied Physics, vol. 26, pp. 182185, 1955.

[19] H. F. Willis, "A formula for expanding an integral as a series," Philosophical Magazine, vol. 39, pp. 455-459, 1948.

[20] C. J. Tranter, Integral Transform in Mathematical Physics, Mathuen, 1966.

[21] H. Ding, W. Chen, and L. Zhang, Elasticity of Transversely Isotropic Materials, Springer, 2006. 


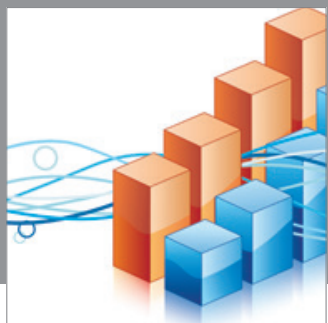

Advances in

Operations Research

mansans

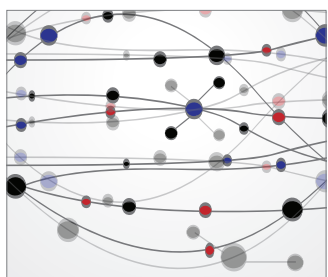

The Scientific World Journal
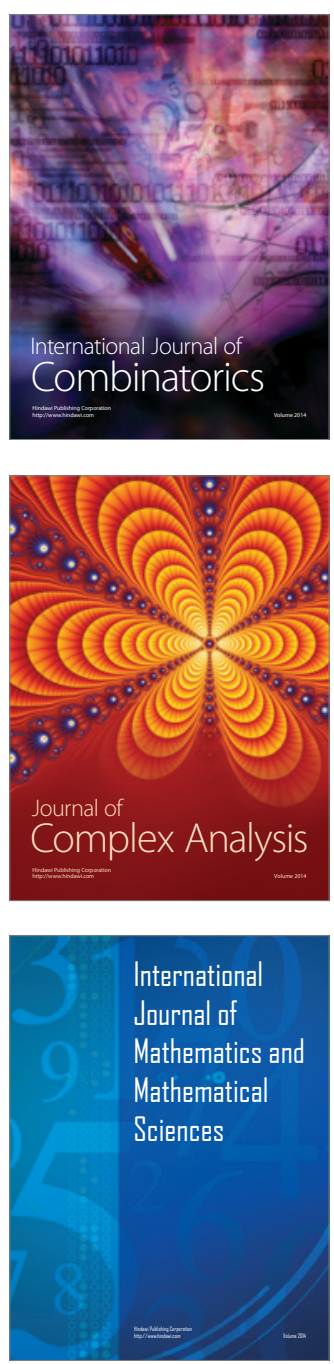
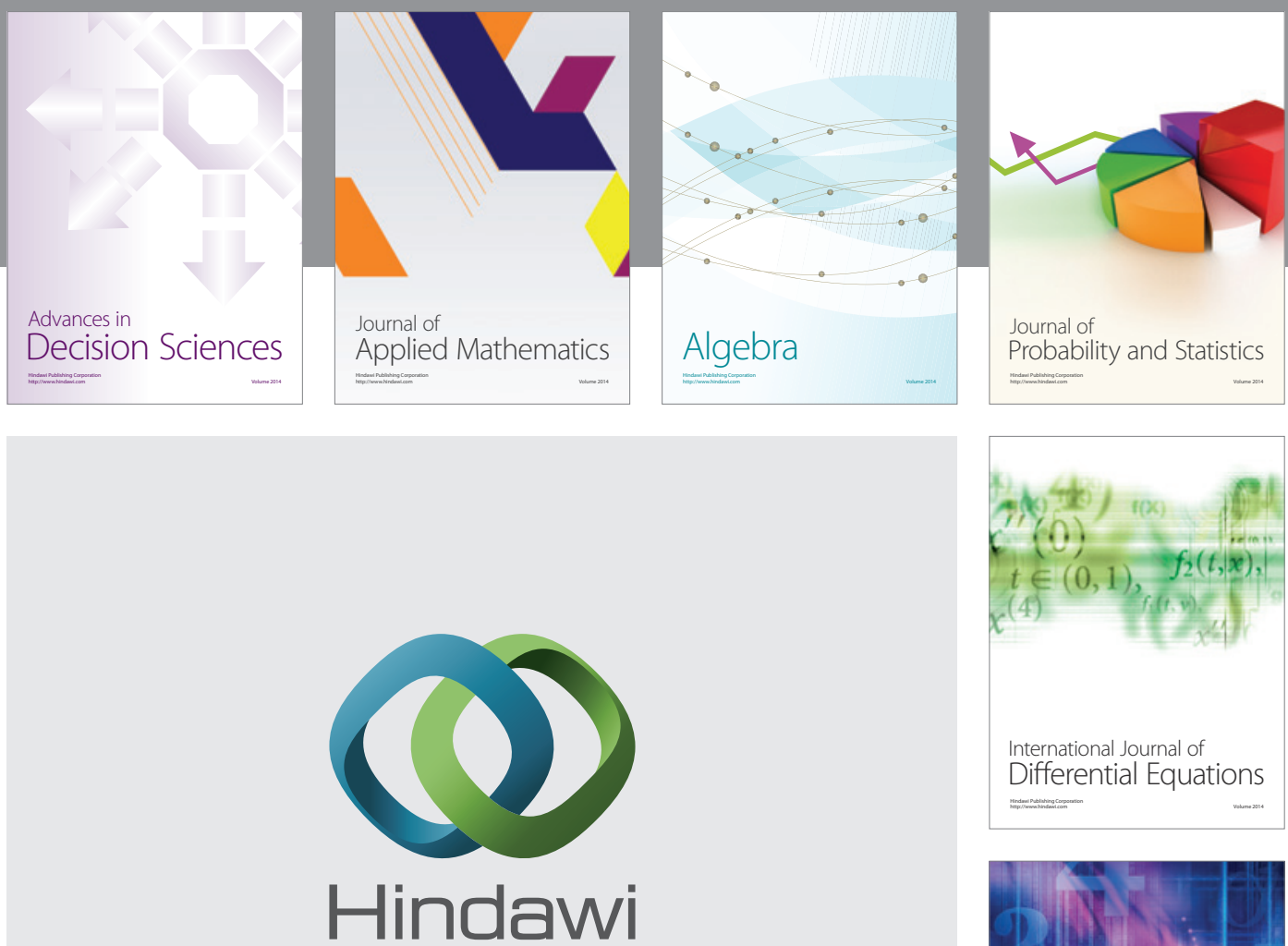

Submit your manuscripts at http://www.hindawi.com
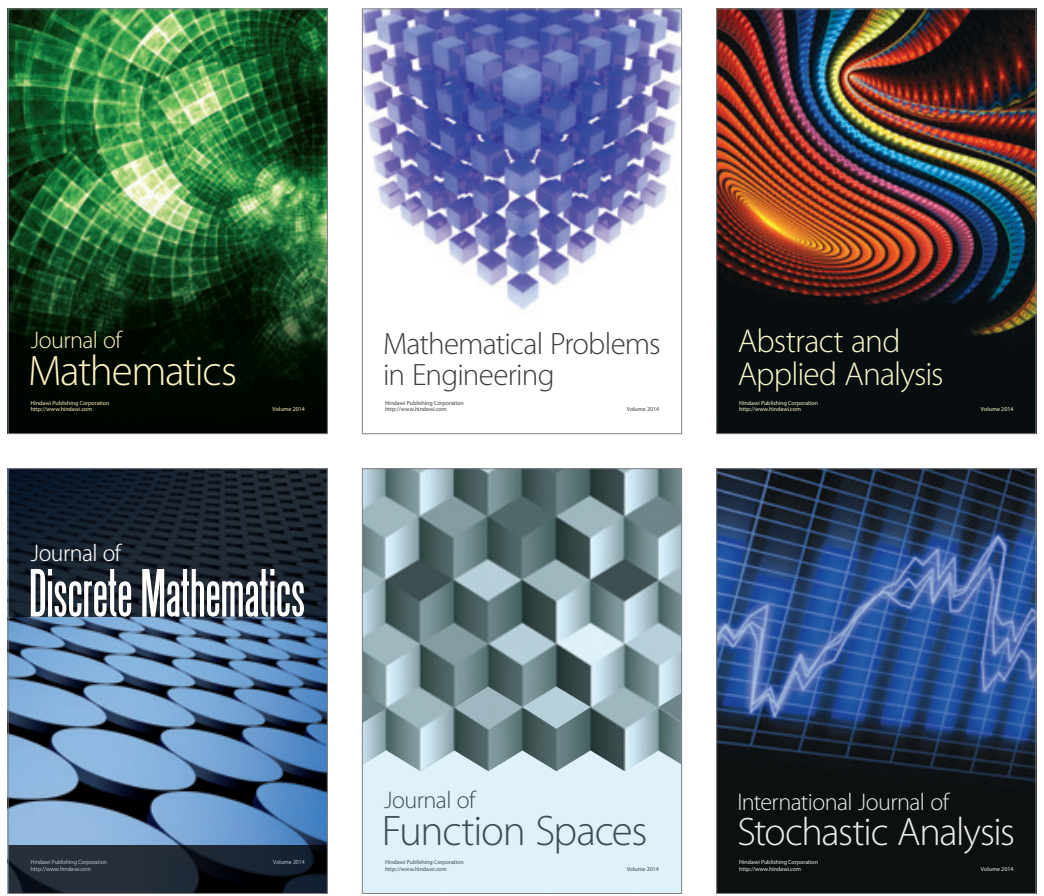

Journal of

Function Spaces

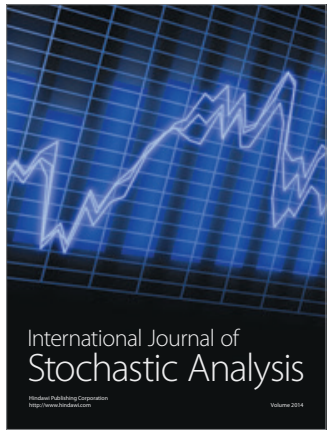

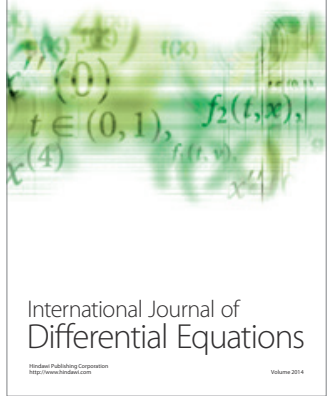
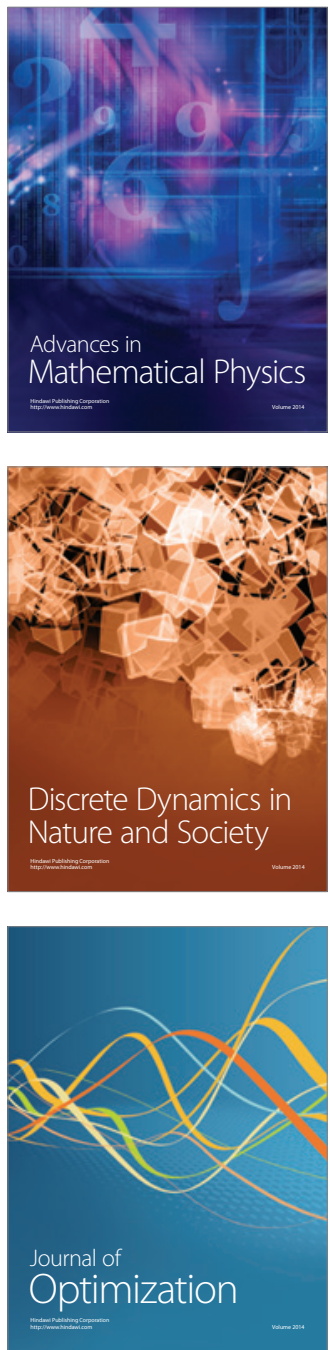